\title{
Identification of candidate protective variants for common diseases and evaluation of their protective potential
}

\author{
Joe M. Butler ${ }^{1}$, Neil Hall ${ }^{2}$, Niro Narendran ${ }^{3}$, Yit C. Yang ${ }^{3}$ and Luminita Paraoan ${ }^{1 *}$ (D)
}

\begin{abstract}
Background: Human polymorphisms with derived alleles that are protective against disease may provide powerful translational opportunities. Here we report a method to identify such candidate polymorphisms and apply it to common non-synonymous SNPs (nsSNPs) associated with common diseases. Our study also sought to establish which of the identified protective nsSNPs show evidence of positive selection, taking this as indirect evidence that the protective variant has a beneficial effect on phenotype. Further, we performed an analysis to quantify the predicted effect of each protective variant on protein function/structure.

Results: An initial analysis of eight SNPs previously identified as associated with age-related macular degeneration $(A M D)$, revealed that two of them have a derived allele that is protective against developing the disease. One is in the complement component 2 gene (C2; E318D) and the other is in the complement factor B gene (CFB; R32Q). Then, combining genomewide ancestral allele information with known common disease-associated nsSNPs from the GWAS catalog, we found 32 additional SNPs which have a derived allele that is disease protective. Out of the total 34 identified candidate protective variants (CPVs), we found that 30 show stronger evidence of positive selection than the protective variant in lipoprotein lipase $(L P L ; S 447 X)$, which has already been translated into gene therapy. Furthermore, 11 of these CPVs have a higher probability of affecting protein structure than the lipoprotein lipase protective variant ( $L P L ;$ S447X).
\end{abstract}

Conclusions: We identify 34 CPVs from the human genome. Diseases they confer protection against include, but are not limited to, type 2 diabetes, inflammatory bowel disease, age-related macular degeneration, multiple sclerosis and rheumatoid arthritis. We propose that those 30 CPVs with evidence of stronger positive selection than the LPL protective variant, may be considered as priority candidates for therapeutic approaches. The next step towards translation will require testing the hypotheses generated by our analyses, specifically whether the CPV arose from a gain-of-function or a loss-of-function mutation.

Keywords: Candidate gene, Protective variants, Common variants, Missense mutation, Gene selection, Age-related macular degeneration, Non-synonymous SNP, Gain of function, Ancestral allele, Type 2 diabetes, Inflammatory bowel disease, Multiple sclerosis and rheumatoid arthritis

\footnotetext{
* Correspondence: Iparaoan@liverpool.ac.uk

'Department of Eye and Vision Science, Institute of Ageing and Chronic

Disease, University of Liverpool, 6 West Derby Street, Liverpool L7 8TX, UK

Full list of author information is available at the end of the article
} 


\section{Background}

Mutations associated with diseases are usually considered to be detrimental to health, increasing the risk of disease. However there are a growing number of reported missense rare mutations shown to be protective, lowering the risk of certain diseases and conditions [1,2]. These are beginning to present valuable therapeutic opportunities [3]. If such a mutation gives rise to a selective advantage, and given enough time, we may expect it to rise in allele frequency to become a common variant in the population. Indeed a well documented example of such a common variant exists, the S447X variant of the lipoprotein lipase (LPL). One phenotypic benefit of this derived allele is its significantly lower risk of cardiovascular diseases and hypertension [4]. Furthermore recently this variant has been utilized therapeutically for the rare condition LPL-deficiency; using a gene therapy approach the derived variant was successfully administered first in mice $[5,6]$ and now in humans as alipogene tiparvovec [7].

In the light of this development, we sought to identify other common protective variants (CPVs) from nonsynonymous (nsSNPs) that have the potential to lead to a translational opportunity. The number of common variants associated with disease continues to grow owing to the endeavour of genome wide association studies (GWAS). The first ever GWAS was carried out for age-related macular degeneration (AMD) [8] and more recently a collaborative GWAS has identified a total of 19 AMD susceptibility loci [9]. These findings led us to begin our search for CPVs in the context of AMD. To ascertain whether a nsSNP is protective (i.e. the respective derived allele is protective) we need to deduce which allele is derived and which is ancestral. For SNPs with a very low minor allele frequency $(<1 \%)$, in a majority of the cases it can be quite safely assumed that the rare allele is the derived allele. However for common variants the assumption that the rare allele is the derived allele can be erroneous. Indeed if a derived allele provided a protective function and gives individuals' a selective advantage one might expect positive selection to sweep it to become the most common allele in the population. Thus for all common variants we must deduce which allele is derived and which is ancestral using the genome alignments with primate species.

The identification of such protective derived variants is of importance to drug development as they represent naturally occurring therapeutic opportunities. There is a high failure rate in pharmaceutical research and development, with less than $5 \%$ of molecules entering Phase I clinical trials actually being approved as safe and effective [10], and around a quarter of those reaching Phase II trials failing due to toxicity [10]. The fact that a protective variant has existed in a part of the human population without adverse effects gives confidence that a therapeutic approach targeting them is less likely to fail in clinical trials.
An understanding of the protein function of a therapeutic candidate is an important factor in helping prioritize and direct further research and ultimately in selecting the most promising targets for a drug development programme [10]. Even before the function and mechanism of action of a protein are characterised through carefully constructed in vitro and/or in vivo molecular biology studies, it is possible to assemble some knowledge of a variant's function using bioinformatics approaches. Specifically, if a protective variant's function gives a selective advantage compared to the ancestral variant then we can use approaches to detect evidence of positive natural selection [11]. Accordingly, we undertook an analysis to establish which of the identified nsSNPs show evidence of positive selection, using this as indirect evidence of the protective variant having a beneficial effect on phenotype through a change in protein function.

A change in protein function can be construed to have arisen from either a gain-of-function or a loss-of-function mutation, thus leading to different translational opportunities. A protective gain-of-function mutation may be harnessed through utilizing the derived allele in a genetherapy approach, such as in the S447X example described above. A protective loss-of-function mutation may be harnessed by therapies that inhibit the function of the ancestral gene [12]. We therefore performed a further bioinformatics analysis to assess whether the identified CPVs were derived through a loss or a gainof-function mutation.

\section{Methods}

\section{Principal logic}

Our work is based on the reasoning that every biallelic SNP is composed of an ancestral allele and a derived allele. If a SNP is associated with a disease then there are two possible situations: (1) either its derived allele is associated with increased risk (being more frequent in cases than controls), or (2) its derived allele is associated with decreased risk (being more frequent in controls than cases). In the latter case, if the SNP is functionally linked to the disease (and is not just a proxy) then it may be termed a protective variant. In this study we sought to identify such protective alleles; to increase the likelihood of it being functional we limited our search to non-synonymous SNPs. We do not confirm the functional impact of these variants, and hence we cannot conclude that they are truly protective. Our study therefore identifies SNPs that they are "potentially" protective and thus we term them as candidate protective variants (CPVs).

The resulting list of CPVs is then subjected to various bioinformatic analyses to initiate the collection of evidence supporting their protective function and thus paving the way for the experimental characterization necessary to confirm whether they are truly protective. 


\section{Ascertaining the ancestral and derived allele of SNPs}

This ancestral allele information was inferred from whole genome alignments of 6 primate species (H. sapiens, $P$. troglodytes, G. gorilla, P. pygmaeus, M. mulatta and $C$. jacchus) released in Ensembl Compara 59 which implemented the Enredo-Pecan-Ortheus (EPO) alignment method [13]. We accessed this information from phase 1 of the 1000 Genomes project [14] by applying the tabix command from the SAM tools package [15] to the relevant chromosome variant call format (VCF) files.

\section{Identifying CPVs from previously reported AMD genetic associations}

To identify CPVs from nsSNPs for AMD we began with the 19 signals reported in the most recent comprehensive meta-analysis [9]. For each signal we used the UCSC genome browser to search nearby genes for nsSNPs associated with AMD [using genome assembly GRCh37/hg19] [16]. Furthermore we only included those nsSNPs which have been validated by meta-analysis.

\section{Identifying CPVs from the GWAS catalog}

To identify CPVs from the raw GWAS catalog we applied seven stages of filtering. Stage 1 involved selecting those associations labelled as missense. Stage 2 involved removing all associations in which the reported risk allele was missing (denoted by a "?" in the catalog). Stage 3 kept only those associations that have an ancestral allele with a high-confidence call. Stage 4 kept only those in which this ancestral allele matches the risk allele i.e. those which have a derived allele that is protective. Stage 5 kept only those associations in which an odds ratio was reported as opposed to a beta regression value (filtering out studies related to continuous traits such as height and heart rate, rather than binomial disease status). For stage 6 we manually scrutinized those studies in which associated genes were oriented on the negative strand, for such genes some studies report the risk allele as the allele on the positive strand whereas others report the coding allele on the negative strand. We removed those associations if it was not clear from the manuscript whether the risk allele referred to the positive or negative strand (note that the reported ancestral allele always refers to the allele on the positive strand). The seventh and final stage involved removing any duplicated associations, i.e. those reported in the catalog by more than one GWAS. The respective numbers of associations remaining after each of the above filter stages are presented in Additional file 1: Table S1.

\section{Measuring selection by calculating integrated haplotype score (iHS)}

For each CPV we estimated its integrated haplotype score (iHS) using the rehh package [17]. This was standardized using the iHS distribution of randomly selected
SNPs whose derived allele frequency matches that (within $2.5 \%)$ of the nsSNP. A negative iHS score indicates that the derived allele has undergone recent positive selection [11] and thus supports the derived allele having a function beneficial for an individual's fitness. For this calculation we considered only common SNPs (maf > 0.01) from the chromosomal region that flank either side of the nsSNP by $100 \mathrm{~kb}$. All SNP data was taken from the 1000 Genomes Project, and by combining all racial groups as a representative sample $(n=1092)$ of the human population.

We sought to increase the confidence of the iHS score by, instead of taking a single iHS value for the single nsSNP, calculating a mean iHS from a set of SNPs that will also be subject to positive selection given that the nsSNP is. This set of SNPs were defined as follows: a SNP was included in the set if satisfied all three following conditions: (i) it was in high LD with the nsSNP (pairwise D' > 0.9) [18]; (ii) it had a derived allele that is inphase with the derived nsSNP allele and (iii) was evolutionary younger than the nsSNP (i.e. had a derived allele frequency less than the derived nsSNP allele frequency).

A graphical way of representing these three conditions was to employ a gene or phylogenetic tree. The set defined above is equivalent to considering all those SNPs that lie on the same branch and on all branches below the nsSNP. For this purpose we implemented the package GENETREE version 9.0 [19]. Note that this program only operates under perfect phylogeny (no recombination) meaning all pairwise D' values need to equal 1 . Thus we necessarily modified our data (where all D' $>0.9$ ) by pruning those rare haplotypes that have occurred from recombination.

\section{Prediction of functional effect of variants}

For $33 \mathrm{CPVs}$, we used PolyPhen-2 [20] to estimate the probability of the mutation being damaging. (One of the initial 34 CPVs (rs20541 in IL13) was not present in the PolyPhen-2 database and therefore is not included in this analysis.) For comparison we also included the probability for the LPL S447X variant. The PolyPhen-2 database (polyphen-2.2.2-whess-2011_12.sqlite) was downloaded using sqlite3. A mean probability was calculated if a mutation had multiple probabilities due to it having different effects on multiple transcripts (splice variants) of the same gene. It is important to note here that the term "damaging" used by PolyPhen- 2 is meant to reflect that the mutation affects protein structure and therefore may affect function either in a gain- or loss-of-function manner. In addition the CPVs were evaluated using the Combined Annotation-Dependent Depletion (CADD) method which objectively integrates many diverse annotations to quantify the pathogenicity of human variants [21]. 


\section{Results}

Identification of two independent CPVs for AMD

We brought together eight AMD-nsSNP associations from six chromosomal loci, each of which had been validated by meta-analysis (Table 1). By ascertaining the ancestral allele for each of these eight nsSNPs we identified that two have a derived allele that is protective; inheriting either one of these alleles reduces the risk of developing AMD. One is the rs9332739 SNP in the complement component 2 gene, C2; the derived allele of which changes its 318th amino acid from glutamic acid to aspartic acid (E318D). The other is the rs641153 SNP in the complement factor B gene, CFB; the derived, protective allele changes its 32nd amino acid from arginine to glutamine (R32Q).

It is interesting that these two nsSNP are in close physical proximity $(\approx 10 \mathrm{~kb}$ apart) but are independent from one another, having low pairwise linkage disequilibrium between them $\left(R^{2}=0.002\right)$. It is not yet known what the level of epistasis between these two variants is upon AMD risk. Assuming no epistasis such that there is no departure from independence of effect sizes, an individual homozygous with both derived alleles (318D/ $318 \mathrm{D}$ and $32 \mathrm{Q} / 32 \mathrm{Q}$ ) is approximately 10 times less likely to develop AMD than an individual homozygous with both ancestral alleles (318E/318E and 32R/32R). This calculation is based upon the homozygote ORs reported in the relevant meta-analysis [22].

Although these SNPs are not in LD with each other, it is important to note that both these SNPs are in high LD with other nsSNPs in the region. Indeed E318D in C2 is in almost complete LD with another nsSNP in the locus, the $\mathrm{L} 9 \mathrm{H}$ variant (rs4151667) in CFB $\left(\mathrm{R}^{2}=0.95\right)$. This is one reason why the AMD association information alone is not sufficient to conclude with certainty that these missense changes are functionally connected to AMD pathogenesis. Further evidence is required to conclude this.

\section{AMD CPVs show stronger evidence of recent positive selection than S447X in LPL}

The fact that these AMD associated variants simply exist in the human population is testament to them not having fatal consequences or adverse effects. Thus a "natural experiment" has already been performed on these variants to test for adverse effects with zero incidences reported. This increases the appeal of them as candidates for therapeutic research and applicability.

To commence the collection of evidence supporting a functional link between these two nsSNPs and AMD pathogenesis we implemented a bioinformatics approach. Specifically we assessed the extent of recent positive selection around these nsSNPs using the integrated haplotype score (iHS) [11]. If an nsSNP has a derived allele that is protective against disease then a positive selection signal is expected given an increased reproductive fitness of those individuals inheriting such an allele. We used this method to compare the evidence for positive selection of the AMD variants against that of the previously translated LPL S447X variant.

The variant $\mathrm{R} 32 \mathrm{Q}$ in $C F B$ presented a mean $\mathrm{iHS}$ of -0.72 calculated from a SNP set comprising 13 SNPs (Fig. 1a and f). For comparison, the mean iHS pertaining to the S447X in $L P L$ was -0.03 , calculated from a set of 28 SNPs (Fig. 1d and $\mathrm{f}$ ). The more negative mean iHS of R32Q in $C F B$ suggested it has undergone more positive selection than S447X in LPL. Considering that S447X has a validated beneficial functional consequence we provisionally concluded that the $C F B \mathrm{R} 32 \mathrm{Q}$ variant must also have a beneficial functional consequence. Inheriting the $Q$ allele appears to have provided a selective advantage and increases an individual's reproductive fitness. Similarly we found the E318D variant in $C 2$ also gives rise to a more negative mean iHS than S447X in LPL. The SNP set consisting of 9 SNPs (Fig. 1b) gives a mean iHS of -0.55 (Fig. 1f). This provided support that this locus has undergone more recent positive selection than $L P L$ S447X, implicating a potentially advantageous function with respect to an individual's fitness.

We repeated this analysis using only those individuals with European ancestry from the 1000 genomes project $(n=379)$. As with the results above using all individuals, both the R32Q and E318D variants gave rise to a more negative mean iHS than S447X. Here however the E318D

Table 1 Non-synonymous SNPs associated with AMD validated by meta-analysis

\begin{tabular}{|c|c|c|c|c|c|}
\hline Position & Locus & nsSNP & & Derived allele & Meta-analysis validation \\
\hline chr1: $196,659,237$ & $\mathrm{CFH}$ & rs1061170:T > C & $\mathrm{Y} 402 \mathrm{H}$ & risk & {$[29]$} \\
\hline chr1: $196,642,233$ & & rs800292:A > G & $162 \mathrm{~V}$ & risk & [30] \\
\hline chr6: $31,903,804$ & $C 2$ & rs9332739:G > C & E318D & protective & {$[22]$} \\
\hline chr6: $31,914,180$ & CFB & rs641153:C > T & R32Q & protective & {$[22]$} \\
\hline chr10: $124,214,448$ & ARMS2 & rs10490924:G > T & A69S & risk & [31] \\
\hline chr19: 6,718,387 & C3 & rs2230199:C > G & $\mathrm{R} 102 \mathrm{G}$ & risk & {$[32]$} \\
\hline chr19: 45,411,941 & $A P O E$ & rs429358:T > C & C112R & risk & [33] \\
\hline chr19: $45,412,079$ & & rs7412:C > T & $\mathrm{R} 158 \mathrm{C}$ & risk & [33] \\
\hline
\end{tabular}


a

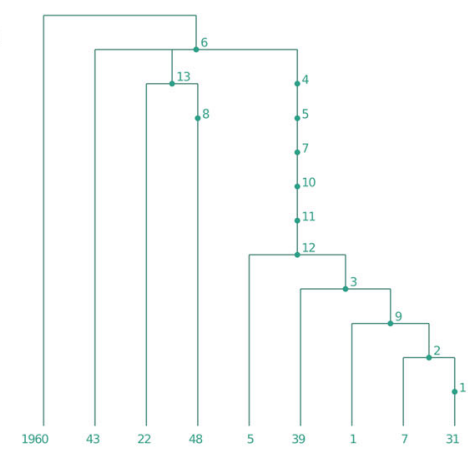

C

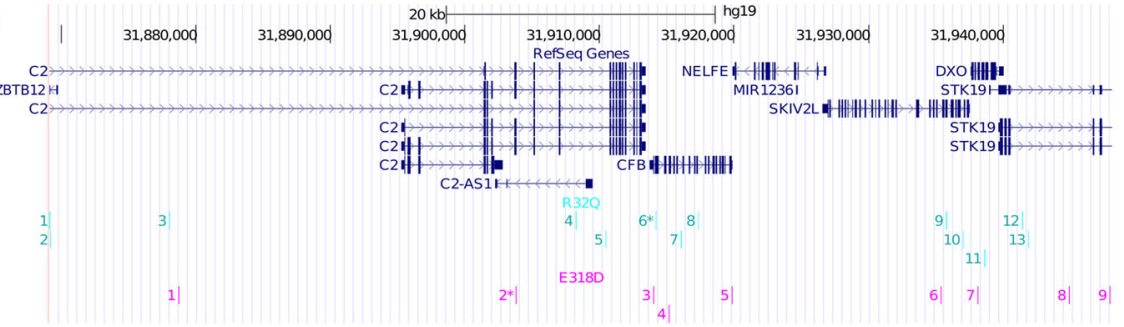

d

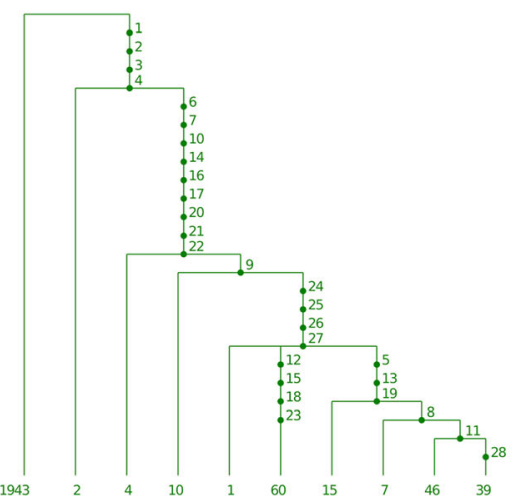

e

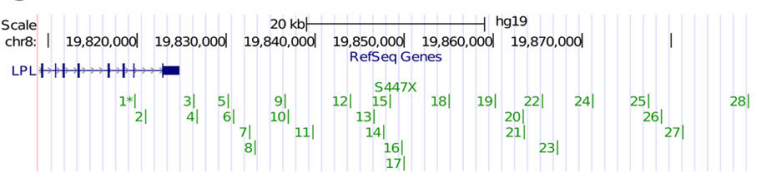

f

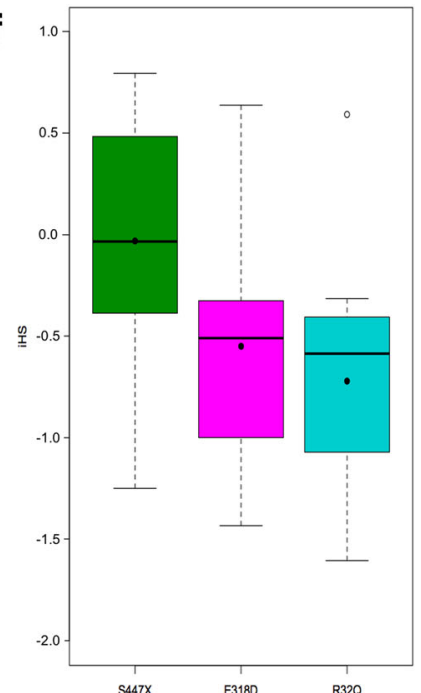

Fig. 1 Integrated haplotype score analysis of the SNP sets corresponding to two AMD candidate protective nsSNPs in C2 and CFB genes and one nsSNP protective against hypertension in LPL. High LD SNP sets, represented as genetic trees, for (a) CFB R32Q, (b) C2 E318D and (d) LPL S447X, where SNPs are numbered on the branches. The numbers at the bottom of each branch represent the number of haplotypes it is comprised of. SNPs on genetic trees are aligned against UCSC genome browser (c) and (e), displaying RefSeq genes in the respective regions (nsSNPs are designated with an asterisk *). The integrated haplotype score (iHS) for each of these SNPs is calculated, represented by the distributions shown in (f) for each nsSNP. Mean iHS is represented by a black circle (•), median by a thick black line and the box represents the central $50 \%$ of the data. An outlier is represented by white circle ( $\left.{ }^{\circ}\right)$

in $C 2$ presented the highest measure of selection (mean iHS $=-0.74$ ), followed by R32Q in CFB (mean iHS $=-0.33)$.

\section{Identification of CPVs for other common diseases}

Next we applied the same reasoning to search the entire GWAS catalog for CPVs and identified a further 32 such variants, in addition to the two associated with AMD (Table 2 and Fig. 2). Out of the total 34 CPVs, 30 gave rise to a more negative mean iHS than $L P L$ S447X. Four of the nsSNPs had a higher mean iHS than LPL S447X, and also had a positive iHS value suggesting that their derived alleles have been subject to negative selection. Taken together the number of CPVs with a mean iHS less than zero $(\mathrm{k}=30)$ is significantly greater than expected by chance $\left(P=2.7 \times 10^{-6}\right.$; Binomial $(34,0.5)$ suggesting that overall, the derived allele of a CPV is more likely to have undergone positive selection than negative selection. 
Table 2 Candidate protective nsSNPs

\begin{tabular}{|c|c|c|c|c|c|c|c|c|c|c|c|}
\hline & Disease & Gene & rsID & Chr & Position & Anc & Der & $\mathrm{OR}^{\mathrm{b}}$ & SNPs in high LD set & Mean iHS & GWAS \\
\hline 1 & Multiple sclerosis & IL7R & rs6897932 & 5 & $35,874,575$ & $C$ & $\mathrm{~T}$ & 1.12 & 6 & -1.462 & {$[34]$} \\
\hline 2 & Glaucoma (primary open-angle) & COL11A1 & rs3753841 & 1 & $103,379,918$ & G & A & 1.20 & 22 & -1.270 & {$[35]$} \\
\hline 3 & Type 2 diabetes & SLC30A8 & rs13266634 & 8 & $118,184,783$ & C & $\mathrm{T}$ & 1.16 & 5 & -1.218 & {$[36]^{a}$} \\
\hline 4 & Psoriasis & TYK2 & rs12720356 & 19 & $10,469,975$ & A & C & 1.40 & 1 & -1.197 & {$[37]$} \\
\hline 5 & Acute lymphoblastic leukemia (childhood) & KCNE4 & rs12621643 & 2 & $223,917,983$ & $\mathrm{~T}$ & G & 1.48 & 3 & -1.167 & {$[38]$} \\
\hline 6 & Ulcerative colitis & IL17REL & rs5771069 & 22 & $50,435,480$ & G & A & 1.11 & 10 & -1.121 & [39] \\
\hline 7 & Esophageal cancer and gastric cancer & PLCE1 & rs3765524 & 10 & $96,058,298$ & $\mathrm{~T}$ & C & 1.35 & 23 & -0.897 & {$[40]$} \\
\hline 8 & IgA nephropathy & TNFSF13 & rs3803800 & 17 & $7,462,969$ & A & G & 1.21 & 23 & -0.819 & [41] \\
\hline 9 & Systemic lupus erythematosus & WDFY4 & rs7097397 & 10 & $50,025,396$ & G & A & 1.30 & 18 & -0.767 & {$[42]$} \\
\hline 10 & Esophageal cancer & PLCE1 & rs 2274223 & 10 & $96,066,341$ & G & A & 1.34 & 38 & -0.740 & [43] \\
\hline 11 & AMD & CFB & rs641153 & 6 & $31,914,180$ & G & A & 2.44 & 13 & -0.723 & - \\
\hline 12 & Inflammatory bowel disease & CD6 & rs11230563 & 11 & $60,776,209$ & C & $\mathrm{T}$ & 1.09 & 21 & -0.705 & {$[44]$} \\
\hline 13 & Behcet's disease & KLRC4 & rs2617170 & 12 & $10,560,957$ & $\mathrm{~T}$ & C & 1.28 & 46 & -0.657 & {$[45]$} \\
\hline 14 & Coronary heart disease & $\mathrm{ZC} 3 \mathrm{HC} 1$ & rs11556924 & 7 & $129,663,496$ & C & $\mathrm{T}$ & 1.09 & 6 & -0.649 & {$[46]$} \\
\hline 15 & Type 1 diabetes & TYK2 & rs2304256 & 19 & $10,475,652$ & C & A & 1.16 & 7 & -0.627 & [47] \\
\hline 16 & Obesity & GIPR & rs1800437 & 19 & $46,181,392$ & G & C & 1.1 & 10 & -0.606 & {$[48]$} \\
\hline 17 & AMD & C2 & rs9332739 & 6 & $31,903,804$ & G & C & 1.82 & 9 & -0.551 & - \\
\hline 18 & Type 2 diabetes & PPARG & rs1801282 & 3 & $12,393,125$ & C & G & 1.14 & 11 & -0.537 & [49] \\
\hline 19 & Inflammatory bowel disease & TUBD1 & rs1292053 & 17 & $57,963,537$ & G & A & 1.08 & 41 & -0.498 & {$[44]$} \\
\hline 20 & Migraine & MMP17 & rs6598163 & 12 & $132,325,239$ & G & A & 1.15 & 22 & -0.493 & {$[50]$} \\
\hline 21 & Glaucoma (exfoliation) & LOXL1 & rs3825942 & 15 & $74,219,582$ & G & A & 20.1 & 26 & -0.453 & [51] \\
\hline 22 & Inflammatory bowel disease & IL23R & rs11209026 & 1 & $67,705,958$ & G & A & 2.01 & 2 & -0.410 & {$[52]$} \\
\hline 23 & Multiple sclerosis & MPV17L2 & rs874628 & 19 & $18,304,700$ & A & G & 1.11 & 8 & -0.292 & [53] \\
\hline 24 & Rheumatoid arthritis & RTKN2 & rs3125734 & 10 & $63,958,112$ & $\mathrm{~T}$ & C & 1.20 & 12 & -0.263 & {$[54]$} \\
\hline 25 & Interstitial lung disease & LRRC34 & rs6793295 & 3 & $169,518,455$ & $C$ & $\mathrm{~T}$ & 1.30 & 19 & -0.228 & {$[55]$} \\
\hline 26 & Obesity & $\mathrm{SH} 2 \mathrm{~B} 1$ & rs7498665 & 16 & $28,883,241$ & G & A & 1.07 & 27 & -0.210 & [48] \\
\hline 27 & Breast cancer & ANKLE1 & rs8100241 & 19 & $17,392,894$ & G & A & 1.14 & 11 & -0.185 & {$[56]$} \\
\hline 28 & Systemic lupus erythematosus & BANK1 & rs10516487 & 4 & $102,751,076$ & G & A & 1.38 & 8 & -0.184 & [57] \\
\hline 29 & Ovarian cancer & ANKLE1 & rs2363956 & 19 & $17,394,124$ & $\mathrm{~T}$ & G & 1.10 & 13 & -0.153 & {$[58]$} \\
\hline 30 & Rheumatoid arthritis & NFKBIE & rs2233434 & 6 & $44,232,920$ & G & A & 1.20 & 32 & -0.114 & [59] \\
\hline- & Hypertension & $L P L$ & rs328 & 8 & $19,819,724$ & C & G & - & 28 & -0.031 & - \\
\hline 31 & Type 2 diabetes & WFS1 & rs1801214 & 4 & $6,303,022$ & $\mathrm{~T}$ & C & 1.13 & 8 & 0.314 & {$[60]$} \\
\hline 32 & Type 2 diabetes & THADA & rs7578597 & 2 & $43,732,823$ & $\mathrm{~T}$ & C & 1.15 & 49 & 0.379 & {$[61]$} \\
\hline 33 & Psoriasis & IL13 & rs20541 & 5 & $131,995,964$ & G & A & 1.27 & 8 & 0.872 & {$[62]$} \\
\hline 34 & Prostate cancer & MLPH & rs2292884 & 2 & $238,443,226$ & G & A & 1.14 & 40 & 0.883 & [63] \\
\hline
\end{tabular}

adenotes the nsSNP has been detected by more than one GWAS in the catalog, we only report the first chronologically

${ }^{b} \mathrm{OR}$ for ancestral allele

The nsSNP that had the most negative mean iHS was found in the interleukin 7 receptor (IL7R) gene, for which the ancestral allele codes for threonine and the derived allele codes for isoleucine (T244I). This nsSNP is associated with multiple sclerosis (with the ancestral allele more frequent in cases) and has been validated by meta-analysis [23]. The nsSNP that gave rise to the next most negative mean iHS was found in the collagen 11 alpha 1 (COL11A1) gene, associated with primary open-angle glaucoma. The ancestral allele codes for proline while the derived, protective allele codes for leucine. The third most negative was found in $S L C 30 A 8$ and is associated with Type 2 diabetes. Its ancestral allele codes for arginine whereas the derived, protective allele codes for tryptophan. We repeated this analysis in the sample with European ancestry and comparable mean iHS results were observed. 


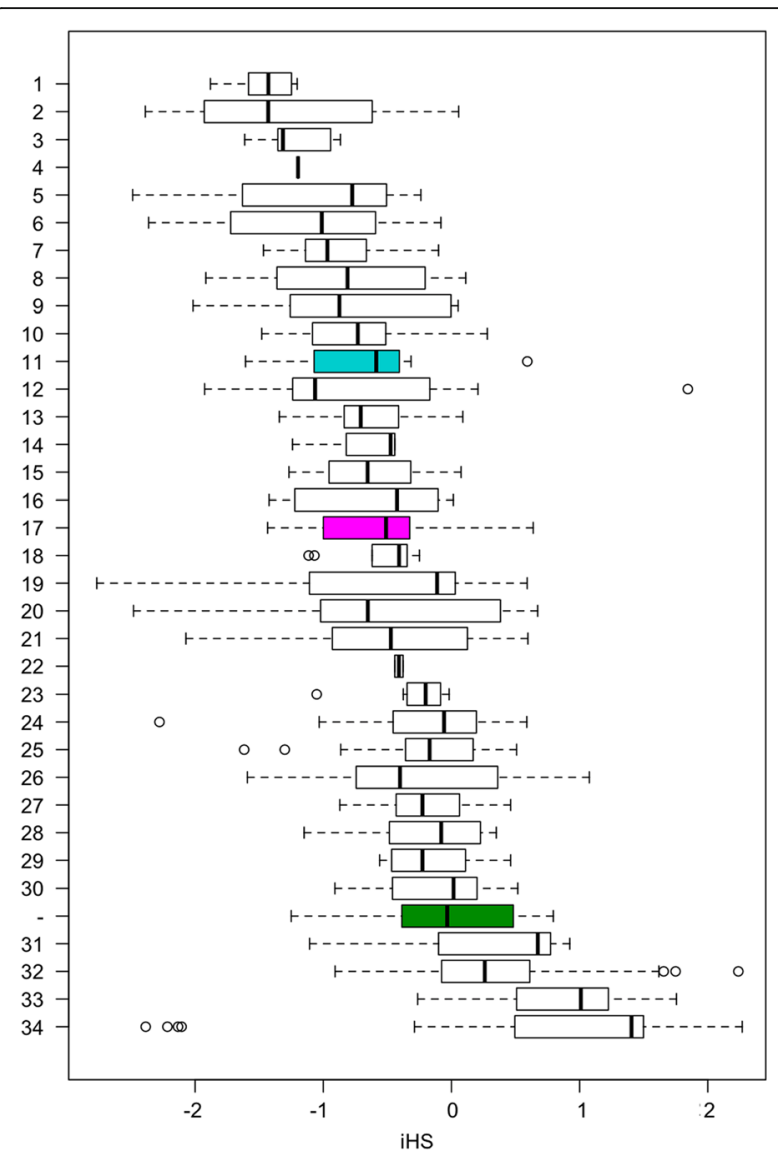

Fig. 2 Integrated haplotype score distributions of the SNP sets corresponding to the 34 candidate protective nsSNPs. High LD SNP sets are computed for each candidate protective nsSNP, from which a distribution of the integrated haplotype scores is calculated and presented as boxplots. The distribution for LPL S447X is shown in green and those for the two AMD nsSNPs in magenta and cyan. The number identifying the nSSNP on the $y$-axis corresponds to the full description in Table 2

For each CPV, the probability of it altering protein structure was calculated using PolyPhen-2 [20]. We found that 11 of the CPVs are predicted to have a higher probability of being damaging than the LPL S447X variant (Fig. 3a). Note that here the word "damaging" refers to altering protein stability and function. We subsequently compared these PolyPhen-2 scores to their CADD scores [21] and find significant positive correlation $(r=0.63$; $P=6.2 \times 10^{-5}$ ) between the two methods (Fig. 3b and Additional file 2: Table S2).

\section{Discussion}

Naturally occurring genetic variants with protective effect against diseases represent a valuable potential resource for translational medicine. Recent advances in gene therapy have realized this by translating the protective effect of one such variant into an effective therapeutic strategy [7]. In this work we identified a total of $34 \mathrm{CPVs}$, having the potential to protect against an array of common diseases. Two of these were identified from an analysis of AMD genetic risk factors, then 32 more were identified through the GWAS catalogue. This is not an exhaustive list because it is unlikely that a recent GWAS has the power to detect all disease-associated nsSNPs, especially rare ones. In addition we only sought those nsSNPs that were directly reported in the GWAS catalogue and thus any causal nsSNP that is captured by a proxy SNP will have been also overlooked by our present method. Linkage disequilibrium incurs a further caveat to this study; for instance, it is possible that a nsSNP at a GWAS signal results in an amino acid change that has no effect on disease risk, but it is in high LD with a non-coding causal SNP elsewhere (e.g. in a regulatory region). Recent evidence does suggest however that coding variants are more likely to be causal than regulatory variants [24]. We also have to consider that there is a chance that a derived mutation may attain a high frequency due to drift, considering that the human race has undergone population bottlenecks [25]. Accordingly we labelled the variants identified in this study as candidate protective variants. In this study we limited our search to nsSNPs directly reported in the GWAS catalog, in order to maximize the likelihood of them being functional. However we note that our list is not a comprehensive catalog of all candidate protective variants; it is also possible that there are functional nsSNPs not reported in the catalog but which are in high LD with non-coding signals (lead SNPs) reported therein.

To advance from being a candidate to being confirmed as truly protective, these variants will need experimental validation based on characterisation of their mechanism of action. Also these candidate protective loci may be subjected to deeper genetic studies, sequencing larger samples of cases and controls to determine if any rare nonsense mutations are associated with the same disease. In this way one can build up an understanding of how different types of functional alterations (e.g. protein-truncation) affect disease risk. However the bioinformatics approaches similar to the one we undertook here build powerful avenues leading to discovery of protective variants for common diseases. We undertook an analysis to determine if the protective function of the derived allele has given it a selective advantage compared to the ancestral allele. We found 30 of the CSVs have a mean iHS that is more negative than the LPL S447X SNP. As the latter has already been successfully translated into gene therapy we conclude that this finding endorses these 30 variants as strong candidates for their protective role against disease. As these variants are still only candidates for being protective, collective further evidence of selection will be informative. The recently developed Singleton Density Score (SDS) [26] is able to detect selection that has occurred more recently than $\mathrm{iHS}$ and therefore would be valuable in 

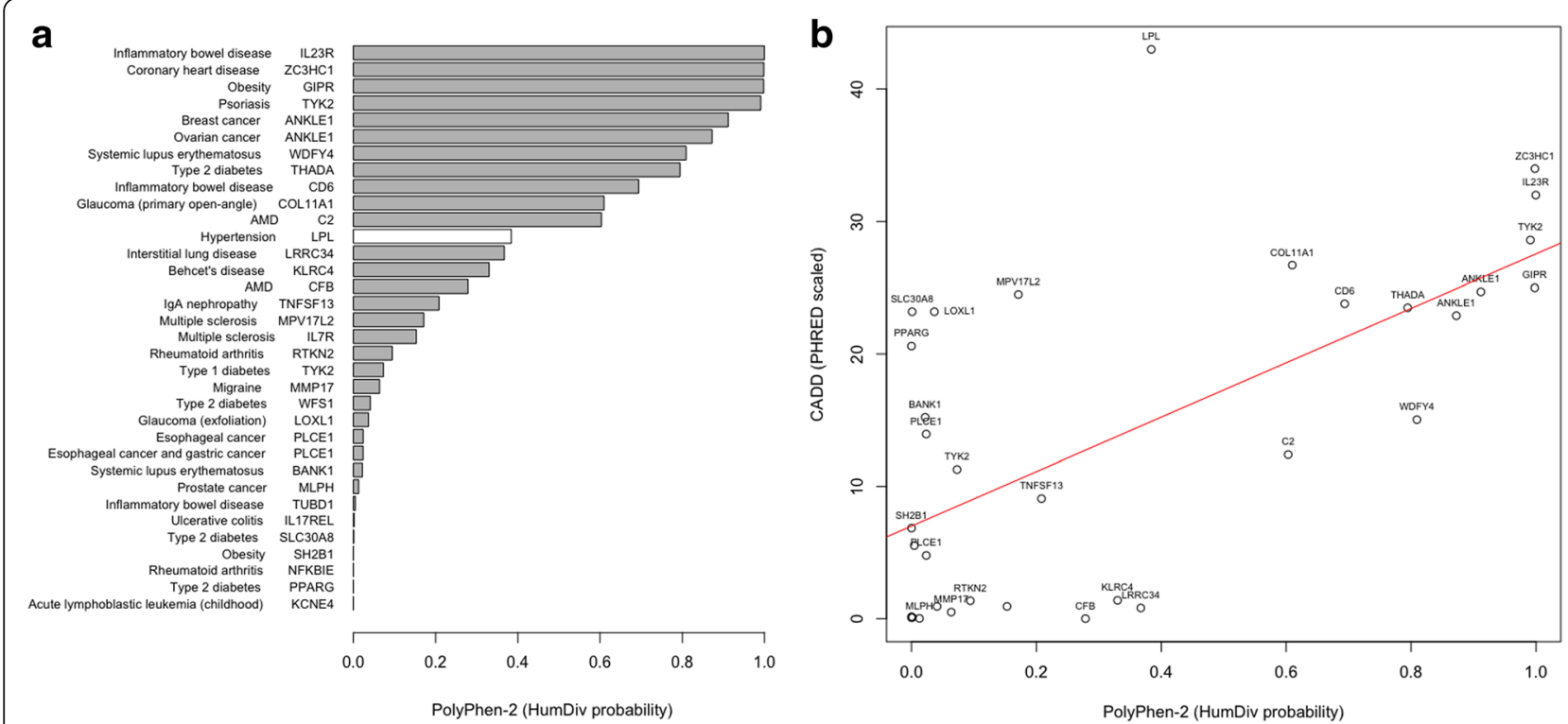

Fig. 3 a Probabilities of the candidate protective variants altering their respective protein function. These probabilities are the Naiive Bayes posterior probability that the missense mutation is damaging based on the PolyPhen-2 HumDiv model. b Positive correlation between PolyPhen-2 and CADD scores

expanding our knowledge of the selection dynamics that have acted upon these CPV loci. Finally with respect to selection we did not find any of the CPVs in a set of 86 regions of selection previously identified in the human genome [27]. However even though our 34 CPVs do not reach genome-wide significance individually, we do find that taken together their iHS scores represent a significant departure towards positive selection $\left(P=2.7 \times 10^{-6}\right)$.

The four CPVs that have a positive mean iHS indicate that they have undergone mild negative selection. Antagonistic pleiotropy could be one explanation for this; although they have a protective function with respect to the studied disease, the derived variant may increase the risk of another disease or present a selective disadvantage. Indeed the two nsSNPs in APOE (see Table 1) make up a set of haplotypes, of which one is known as $\varepsilon 4$ and is well documented to be implicated in the pathogenesis of both AMD and Alzheimer's disease (AD). Whereas the $\varepsilon 4$ allele decreases an individual's AMD risk, it increases AD risk. Thus knowledge of such antagonistic pleiotropy is very important as it represents an obstacle for therapeutic translation; whereas an intervention based on such a variant may achieve a reduction in the risk of developing one disease, it may increase the risk of another. Thus before any variant can be deemed truly protective such pleiotropic factors must be investigated, including those factors that affect selection such as how prevalent are the diseases involved and what are their ages of onset. Because the majority of the CPVs identified here are for relatively late onset diseases, which are not expected to engender strong selective pressures, the derived allele may have undergone positive selection due to providing another fitness advantage in younger individuals.

The two CPVs in $C F B$ and $C 2$, both protective against AMD, are of particular interest in that although they are close in physical distance $(\approx 10 \mathrm{~kb}$ apart $)$ they are in linkage equilibrium with one another. If their protective effects are found to be independent (no epistasis) then an individual homozygous for the derived allele at both sites is about 10 times less likely to develop AMD than an individual homozygous for the ancestral allele at both sites. We also find these SNPs to be of interest because they show evidence of recent positive selection (Fig. 1) although the disease they protect against is a late-onset disease. We speculate therefore that these may also be protective against another disease, or offer another selective advantage. This speculation is backed by the fact that these two nsSNPs are in genes with function in the complement system, a key component of innate immunity.

Also of interest with respect to imminent translational research is the CPV identified in SLCBOA8 with the derived allele predicted to be protective against Type 2 Diabetes (T2D). Recent evidence elsewhere has validated a direct functional role of this gene in T2D pathogenesis [12]. Specifically, deep sequencing of very large samples has confirmed that rare truncating, loss-of-function mutations are protective against $\mathrm{T} 2 \mathrm{D}$. The authors suggested the approach based on inhibiting the protein encoded by this gene as a therapeutic strategy for T2D prevention. If a protective variant is rare then it represents a particularly valuable therapeutic opportunity because a large fraction of the population is not subjected to its protective effect. Thus if 
translated, a rare protective variant has the potential to benefit more of the population than if the protective variant was common (assuming those already carrying the protective variant will not experience additional protection). Motivated by this, it will also be worth exploring non-coding rare protective alleles such as the two recently found to decrease AMD risk at the CFH locus (rs191281603 and rs148553336) [28].

Bringing together the research on SLC3OA8 and LPL we highlight the fact that two distinct mechanisms, based on loss-of-function and gain-of-function mutation respectively, can both generate a protective variant. Determining which mechanism gave rise to a protective variant will be consequential in choosing the type of translational approach. As suggested for $S L C 30 A 8$ with respect to T2D, if loss-of-function variants are observed to be protective then a therapeutic strategy aimed at inhibiting the gene product is appropriate. On the other hand, if gain-of-function variants are observed to be protective then a gene-therapy approach is likely to be the most appropriate, as employed by the $L P L$ S447X-based therapy. It is also conceivable to administer the gain-of-function protein to the site of pathogenesis.

\section{Conclusions}

This study presents the first systematic analysis of the human genome undertaken to identify CPVs. We identified 34 such variants; the diseases they confer protection against include, but are not limited to T2D, inflammatory bowel disease, multiple sclerosis and rheumatoid arthritis. We propose that these represent highly promising translational targets, potentially accelerating the pathways to novel therapeutic strategies (e.g. gene therapy). Importantly, as the CPVs are naturally occurring they may substantially improve the effectiveness and safety of such therapeutic strategies.

To investigate the evolutionary selection upon these variants we developed a new method based on the previously proposed integrated haplotype score (iHS). Applying this we found that $30 \mathrm{CPVs}$ show evidence of stronger positive selection than the LPL S447X protective variant, which has already been translated into gene therapy. We also carried out a bioinformatics analysis to ascertain the type and size of the effect that the CPV has on protein function. We found that 11 of the $34 \mathrm{CPVs}$ are predicted to have a higher probability of being damaging than the LPL S447X mutation. The next step towards translation will require molecular experimental approaches to test the predictions generated by our analyses. This knowledge will in turn inform the type of translational approach to take: either a gene therapybased approach (if protection is granted by a gain of beneficial function mutation) or molecular inhibition strategies (if protection is afforded by a loss of specific function).

\section{Additional files}

Additional file 1: Table S1. Summary of filtering stages performed on GWAS catalog. (DOCX 59 kb)

Additional file 2: Table S2. Comparison of methods (PolyPhen-2 and CADD) to predict functional effect of CPVs. (DOCX $92 \mathrm{~kb}$ )

\begin{abstract}
Abbreviations
AD: Alzheimer's Disease; AMD: age-related macular degeneration; ApoE: Apolipoprotein E; CADD: Combined Annotation-Dependent Depletion; CFB: complement factor B; CFH: complement factor H; COL11A1: collagen 11 alpha 1; CPV: candidate protective variants; EPO: Enredo-Pecan-Ortheus; GWAS: genome-wide association study; iHS: integrated haplotype score; IL7R: interleukin 7 receptor; kb: kilobase; LD: linkage disequilibrium; LPL: lipoprotein lipase; nsSNP: non-synonymous single nucleotide polymorphism; SDS: Singleton Density Score; T2D: type 2 diabetes; UCSC: University of California at Santa Cruz
\end{abstract}

\section{Acknowledgements}

The authors gratefully acknowledge the support from R \& D Royal Wolverhampton NHS Trust, Wolverhampton, UK.

Funding

No funding was obtained for this study.

Availability of data and materials

The datasets analysed during the current study are available from the GWAS Catalog, www.ebi.ac.uk/gwas/ (accessed 28/12/2013) and for the ancestral allele information from the 1000 Genomes Project ftp://ftp.1000genomes.ebi.ac.uk/ vol1/ftp/.

Authors' contributions

$J M B, L P$ and $N H$ designed the study; JMB acquired the data and carried out the analysis; all authors contributed to the analysis and interpretation of data. All authors were involved in drafting the manuscript or revising it critically for important intellectual content, gave final approval of the version to be published and agreed to be accountable for all aspects related to the accuracy or integrity of any part of the work.

Ethics approval and consent to participate

No human or animal material has been directly used, as the study has used publicly available datasets.

Consent for publication

Not applicable.

Competing interests

The authors declare that they have no competing interests.

\section{Publisher's Note}

Springer Nature remains neutral with regard to jurisdictional claims in published maps and institutional affiliations.

\section{Author details}

${ }^{1}$ Department of Eye and Vision Science, Institute of Ageing and Chronic Disease, University of Liverpool, 6 West Derby Street, Liverpool L7 8TX, UK. ${ }^{2}$ The Earlham Institute, Norwich Research Park, Norwich NR4 7UH, UK. ${ }^{3}$ Department of Ophthalmology, The Royal Wolverhampton NHS Trust, New Cross Hospital, Wolverhampton WV10 0QP, UK.

Received: 24 November 2016 Accepted: 27 July 2017

Published online: 03 August 2017

\section{References}

1. Steinthorsdottir V, Thorleifsson G, Sulem P, Helgason H, Grarup N, Sigurdsson A, Helgadottir HT, Johannsdottir H, Magnusson OT, Gudjonsson SA, et al. Identification of low-frequency and rare sequence variants associated with elevated or reduced risk of type 2 diabetes. Nat Genet. 2014; 
2. Cohen J, Pertsemlidis A, Kotowski IK, Graham R, Garcia CK, Hobbs HH. Low $\mathrm{LDL}$ cholesterol in individuals of African descent resulting from frequent nonsense mutations in PCSK9. Nat Genet. 2005;37(2):161-5.

3. Sullivan D, Olsson AG, Scott R, Kim JB, Xue A, Gebski V, Wasserman SM, Stein EA. Effect of a monoclonal antibody to PCSK9 on low-density lipoprotein cholesterol levels in statin-intolerant patients: the GAUSS randomized trial. JAMA. 2012;308(23):2497-506.

4. Niu WQ, Qi Y. Meta-based association of the lipoprotein lipase gene S447X variant with hypertension and blood pressure variation. J Hum Hypertens. 2011;25(6):383-90.

5. Ross CJ, Liu G, Kuivenhoven JA, Twisk J, Rip J, van Dop W, Excoffon KJ, Lewis SM, Kastelein JJ, Hayden MR. Complete rescue of lipoprotein lipasedeficient mice by somatic gene transfer of the naturally occurring LPLS447X beneficial mutation. Arterioscler Thromb Vasc Biol. 2005;25(10):2143-50.

6. Rader DJ. Gain-of-function mutations and therapeutic implications - lipoprotein lipase S447X to the rescue. Arterioscl Throm Vas. 2005;25(10):2018-9.

7. Gaudet D, Methot J, Dery S, Brisson D, Essiembre C, Tremblay G, Tremblay K, de Wal J, Twisk J, van den Bulk N, et al. Efficacy and long-term safety of alipogene tiparvovec (AAV1-LPLS447X) gene therapy for lipoprotein lipase deficiency: an open-label trial. Gene Ther. 2013;20(4):361-9.

8. Klein RJ, Zeiss C, Chew EY, Tsai JY, Sackler RS, Haynes C, Henning AK, SanGiovanni JP, Mane SM, Mayne ST, et al. Complement factor H polymorphism in agerelated macular degeneration. Science. 2005;308(5720):385-9.

9. Fritsche LG, Chen W, Schu M, Yaspan BL, Yu Y, Thorleifsson G, Zack DJ, Arakawa $S$, Cipriani V, Ripke $S$, et al. Seven new loci associated with agerelated macular degeneration. Nat Genet. 2013;45(4):433-9.

10. Plenge RM, Scolnick EM, Altshuler D. Validating therapeutic targets through human genetics. Nat Rev Drug Discov. 2013;12(8):581-94.

11. Voight BF, Kudaravalli S, Wen X, Pritchard JK. A map of recent positive selection in the human genome. PLoS Biol. 2006;4(3):e72.

12. Flannick J, Thorleifsson G, Beer NL, Jacobs SB, Grarup N, Burtt NP, Mahajan A, Fuchsberger C, Atzmon G, Benediktsson R, et al. Loss-of-function mutations in SLC30A8 protect against type 2 diabetes. Nat Genet. 2014;46(4):357-63.

13. Paten B, Herrero J, Beal K, Fitzgerald S, Birney E. Enredo and pecan: genome-wide mammalian consistency-based multiple alignment with paralogs. Genome Res. 2008;18(11):1814-28.

14. Genomes Project C, Abecasis GR, Altshuler D, Auton A, Brooks LD, Durbin RM, Gibbs RA, Hurles ME, GA MV. A map of human genome variation from population-scale sequencing. Nature. 2010;467(7319):1061-73.

15. Li H, Handsaker B, Wysoker A, Fennell T, Ruan J, Homer N, Marth G, Abecasis $\mathrm{G}$, Durbin R. The sequence alignment/map format and SAMtools. Bioinformatics. 2009;25(16):2078-9.

16. Kent WJ, Sugnet CW, Furey TS, Roskin KM, Pringle TH, Zahler AM, Haussler D. The human genome browser at UCSC. Genome Res. 2002;12(6):996-1006.

17. Gautier M, Vitalis R. rehh: an R package to detect footprints of selection in genome-wide SNP data from haplotype structure. Bioinformatics. 2012;28(8): 1176-7.

18. Lewontin RC. The interaction of selection and linkage. I General Considerations; Heterotic Models. Genetics. 1964;49(1):49-67.

19. Bahlo M, Griffiths RC. Inference from gene trees in a subdivided population. Theor Popul Biol. 2000;57(2):79-95.

20. Adzhubei IA, Schmidt S, Peshkin L, Ramensky VE, Gerasimova A, Bork P, Kondrashov AS, Sunyaev SR. A method and server for predicting damaging missense mutations. Nat Methods. 2010;7(4):248-9.

21. Kircher M, Witten DM, Jain P, O'Roak BJ, Cooper GM, Shendure J. A general framework for estimating the relative pathogenicity of human genetic variants. Nat Genet. 2014;46(3):310-5.

22. Thakkinstian A, McEvoy M, Chakravarthy U, Chakrabarti S, McKay GJ, Ryu E, Silvestri G, Kaur I, Francis P, Iwata T, et al. The association between complement component 2/complement factor B polymorphisms and age-related macular degeneration: a HuGE review and meta-analysis. Am J Epidemiol. 2012;176(5):361-72.

23. Zhang R, Duan L, Jiang Y, Zhang X, Sun P, Li J, Zhang M, Tang G, Wang X, Li X. Association between the IL7R T244I polymorphism and multiple sclerosis: a meta-analysis. Mol Biol Rep. 2011;38(8):5079-84.

24. Kichaev G, Yang WY, Lindstrom S, Hormozdiari F, Eskin E, Price AL, Kraft P, Pasaniuc B. Integrating functional data to prioritize causal variants in statistical fine-mapping studies. PLoS Genet. 2014;10(10):e1004722.

25. Li H, Durbin R. Inference of human population history from individual whole-genome sequences. Nature. 2011;475(7357):493-6.
26. Field Y, Boyle EA, Telis N, Gao Z, Gaulton KJ, Golan D, Yengo L, Rocheleau G, Froguel P, McCarthy Ml, et al. Detection of human adaptation during the past 2000 years. Science. 2016;354(6313):760-4.

27. Grossman SR, Andersen KG, Shlyakhter I, Tabrizi S, Winnicki S, Yen A, Park DJ, Griesemer D, Karlsson EK, Wong SH, et al. Identifying recent adaptations in large-scale genomic data. Cell. 2013;152(4):703-13.

28. Fritsche LG, Igl W, Bailey JN, Grassmann F, Sengupta S, Bragg-Gresham JL, Burdon KP, Hebbring SJ, Wen C, Gorski M, et al. A large genome-wide association study of age-related macular degeneration highlights contributions of rare and common variants. Nat Genet. 2016;48(2):134-43.

29. Sofat R, Casas JP, Webster AR, Bird AC, Mann SS, Yates JR, Moore AT, Sepp T, Cipriani V, Bunce $\mathrm{C}$, et al. Complement factor $\mathrm{H}$ genetic variant and agerelated macular degeneration: effect size, modifiers and relationship to disease subtype. Int J Epidemiol. 2012;41(1):250-62.

30. Yuan D, Yang Q, Liu X, Yuan S, Xie P, Liu Q. Complement factor H Val62lle variant and risk of age-related macular degeneration: a meta-analysis. Mol Vis. 2013;19:374-83.

31. Tong Y, Liao J, Zhang Y, Zhou J, Zhang H, Mao M. LOC387715/HTRA1 gene polymorphisms and susceptibility to age-related macular degeneration: a HuGE review and meta-analysis. Mol Vis. 2010;16:1958-81.

32. Thakkinstian A, McKay GJ, McEvoy M, Chakravarthy U, Chakrabarti S, Silvestri G, Kaur I, Li X, Attia J. Systematic review and meta-analysis of the association between complement component 3 and age-related macular degeneration: a HuGE review and meta-analysis. Am J Epidemiol. 2011; 173(12):1365-79.

33. McKay GJ, Patterson CC, Chakravarthy U, Dasari S, Klaver CC, Vingerling JR, Ho L, de Jong PT, Fletcher AE, Young IS, et al. Evidence of association of APOE with age-related macular degeneration: a pooled analysis of 15 studies. Hum Mutat. 2011;32(12):1407-16.

34. De Jager PL, Jia X, Wang J, de Bakker PI, Ottoboni L, Aggarwal NT, Piccio L, Raychaudhuri S, Tran D, Aubin C, et al. Meta-analysis of genome scans and replication identify CD6, IRF8 and TNFRSF1A as new multiple sclerosis susceptibility loci. Nat Genet. 2009;41(7):776-82.

35. Vithana EN, Khor CC, Qiao C, Nongpiur ME, George R, Chen LJ, Do T, Abu-Amero K, Huang CK, Low S, et al. Genome-wide association analyses identify three new susceptibility loci for primary angle closure glaucoma. Nat Genet. 2012;44(10):1142-6.

36. Bouatia-Naji N, Bonnefond A, Cavalcanti-Proenca C, Sparso T, Holmkvist J, Marchand M, Delplanque J, Lobbens S, Rocheleau G, Durand E, et al. A variant near MTNR1B is associated with increased fasting plasma glucose levels and type 2 diabetes risk. Nat Genet. 2009;41(1):89-94.

37. Strange A, Capon F, Spencer CC, Knight J, Weale ME, Allen MH, Barton A, Band G, Bellenguez C, Bergboer JG, et al. A genome-wide association study identifies new psoriasis susceptibility loci and an interaction between HLA-C and ERAP1. Nat Genet. 2010;42(11):985-90.

38. Trevino LR, Yang W, French D, Hunger SP, Carroll WL, Devidas M, Willman C, Neale G, Downing J, Raimondi SC, et al. Germline genomic variants associated with childhood acute lymphoblastic leukemia. Nat Genet. 2009;41(9):1001-5.

39. Anderson CA, Boucher G, Lees CW, Franke A, D'Amato M, Taylor KD, Lee JC, Goyette P, Imielinski M, Latiano A, et al. Meta-analysis identifies 29 additional ulcerative colitis risk loci, increasing the number of confirmed associations to 47. Nat Genet. 2011;43(3):246-52.

40. Abnet CC, Freedman ND, Hu N, Wang Z, Yu K, Shu XO, Yuan JM, Zheng W, Dawsey SM, Dong LM, et al. A shared susceptibility locus in PLCE1 at 10q23 for gastric adenocarcinoma and esophageal squamous cell carcinoma. Nat Genet. 2010;42(9):764-7.

41. Yu XQ, Li M, Zhang H, Low HQ, Wei X, Wang JQ, Sun LD, Sim KS, Li Y, Foo JN, et al. A genome-wide association study in Han Chinese identifies multiple susceptibility loci for IgA nephropathy. Nat Genet. 2012;44(2):178-82.

42. Yang W, Shen N, Ye DQ, Liu Q, Zhang Y, Qian XX, Hirankarn N, Ying D, Pan HF, Mok CC, et al. Genome-wide association study in Asian populations identifies variants in ETS1 and WDFY4 associated with systemic lupus erythematosus. PLoS Genet. 2010;6(2):e1000841.

43. Wu C, Hu Z, He Z, Jia W, Wang F, Zhou Y, Liu Z, Zhan Q, Liu Y, Yu D, et al. Genome-wide association study identifies three new susceptibility loci for esophageal squamous-cell carcinoma in Chinese populations. Nat Genet. 2011;43(7):679-84

44. Jostins L, Ripke S, Weersma RK, Duerr RH, McGovern DP, Hui KY, Lee JC, Schumm LP, Sharma $Y$, Anderson CA, et al. Host-microbe interactions have shaped the genetic architecture of inflammatory bowel disease. Nature. 2012;491(7422):119-24. 
45. Kirino Y, Bertsias G, Ishigatsubo Y, Mizuki N, Tugal-Tutkun I, Seyahi E, Ozyazgan Y, Sacli FS, Erer B, Inoko H, et al. Genome-wide association analysis identifies new susceptibility loci for Behcet's disease and epistasis between HLA-B*51 and ERAP1. Nat Genet. 2013:45(2):202-7.

46. Schunkert H, Konig IR, Kathiresan S, Reilly MP, Assimes TL, Holm H, Preuss M, Stewart AF, Barbalic M, Gieger C, et al. Large-scale association analysis identifies 13 new susceptibility loci for coronary artery disease. Nat Genet. 2011:43(4):333-8.

47. Wallace C, Smyth DJ, Maisuria-Armer M, Walker NM, Todd JA, Clayton DG. The imprinted DLK1-MEG3 gene region on chromosome 14q32.2 alters susceptibility to type 1 diabetes. Nat Genet. 2010;42(1):68-71.

48. Berndt SI, Gustafsson S, Magi R, Ganna A, Wheeler E, Feitosa MF, Justice AE, Monda KL, Croteau-Chonka DC, Day FR, et al. Genome-wide meta-analysis identifies 11 new loci for anthropometric traits and provides insights into genetic architecture. Nat Genet. 2013;45(5):501-12.

49. Scott LJ, Mohlke KL, Bonnycastle LL, Willer CJ, Li Y, Duren WL, Erdos MR, Stringham HM, Chines PS, Jackson AU, et al. A genome-wide association study of type 2 diabetes in Finns detects multiple susceptibility variants. Science. 2007;316(5829):1341-5.

50. Freilinger T, Anttila V, de Vries B, Malik R, Kallela M, Terwindt GM, PozoRosich P, Winsvold B, Nyholt DR, van Oosterhout WP, et al. Genome-wide association analysis identifies susceptibility loci for migraine without Aura. Nat Genet. 2012;44(7):777-82.

51. Thorleifsson G, Magnusson KP, Sulem P, Walters GB, Gudbjartsson DF, Stefansson H, Jonsson T, Jonasdottir A, Stefansdottir G, Masson G, et al. Common sequence variants in the LOXL1 gene confer susceptibility to exfoliation glaucoma. Science. 2007;317(5843):1397-400.

52. Duerr RH, Taylor KD, Brant SR, Rioux JD, Silverberg MS, Daly MJ, Steinhart AH, Abraham C, Regueiro M, Griffiths A, et al. A genome-wide association study identifies IL23R as an inflammatory bowel disease gene. Science. 2006;314(5804):1461-3.

53. Sawcer S, Hellenthal G, Pirinen M, Spencer CC, Patsopoulos NA, Moutsianas L, Dilthey A, Su Z, Freeman C, Hunt SE, et al. Genetic risk and a primary role for cellmediated immune mechanisms in multiple sclerosis. Nature. 2011;476(7359):214-9.

54. Myouzen K, Kochi Y, Okada Y, Terao C, Suzuki A, Ikari K, Tsunoda T, Takahashi A, Kubo M, Taniguchi A, et al. Functional variants in NFKBIE and RTKN2 involved in activation of the NF-kappaB pathway are associated with rheumatoid arthritis in Japanese. PLoS Genet. 2012;8(9):e1002949.

55. Fingerlin TE, Murphy E, Zhang W, Pelito AL, Brown KK, Steele MP, Loyd JE, Cosgrove GP, Lynch D, Groshong S, et al. Genome-wide association study identifies multiple susceptibility loci for pulmonary fibrosis. Nat Genet. 2013; 45(6):613-20.

56. Siddiq A, Couch FJ, Chen GK, Lindstrom S, Eccles D, Millikan RC, Michailidou K, Stram DO, Beckmann L, Rhie SK, et al. A meta-analysis of genome-wide association studies of breast cancer identifies two novel susceptibility loci at 6q14 and 20q11. Hum Mol Genet. 2012;21(24):5373-84.

57. Kozyrev SV, Abelson AK, Wojcik J, Zaghlool A, Linga Reddy MV, Sanchez E, Gunnarsson I, Svenungsson E, Sturfelt G, Jonsen A, et al. Functional variants in the B-cell gene BANK1 are associated with systemic lupus erythematosus. Nat Genet. 2008;40(2):211-6.

58. Bolton KL, Tyrer J, Song H, Ramus SJ, Notaridou M, Jones C, Sher T, GentryMaharaj A, Wozniak E, Tsai YY, et al. Common variants at 19p13 are associated with susceptibility to ovarian cancer. Nat Genet. 2010;42(10):880-4.

59. Okada Y, Terao C, Ikari K, Kochi Y, Ohmura K, Suzuki A, Kawaguchi T, Stahl EA, Kurreeman FA, Nishida $N$, et al. Meta-analysis identifies nine new loci associated with rheumatoid arthritis in the Japanese population. Nat Genet. 2012;44(5):511-6.

60. Voight BF, Scott $\sqcup$, Steinthorsdottir V, Morris AP, Dina C, Welch RP, Zeggini E, Huth C, Aulchenko YS, Thorleifsson G, et al. Twelve type 2 diabetes susceptibility loci identified through large-scale association analysis. Nat Genet. 2010;42(7):579-89.

61. Zeggini E, Scott $L$, Saxena R, Voight BF, Marchini JL, Hu T, de Bakker PI, Abecasis GR, Almgren P, Andersen G, et al. Meta-analysis of genome-wide association data and large-scale replication identifies additional susceptibility loci for type 2 diabetes. Nat Genet. 2008;40(5):638-45.

62. Nair RP, Duffin KC, Helms C, Ding J, Stuart PE, Goldgar D, Gudjonsson JE, Li Y, Tejasvi T, Feng BJ, et al. Genome-wide scan reveals association of psoriasis with IL-23 and NF-kappaB pathways. Nat Genet. 2009;41(2):199-204.

63. Schumacher FR, Berndt SI, Siddiq A, Jacobs KB, Wang Z, Lindstrom S, Stevens VL, Chen C, Mondul AM, Travis RC, et al. Genome-wide association study identifies new prostate cancer susceptibility loci. Hum Mol Genet. 2011;20(19):3867-75.

\section{Submit your next manuscript to BioMed Central and we will help you at every step:}

- We accept pre-submission inquiries

- Our selector tool helps you to find the most relevant journal

- We provide round the clock customer support

- Convenient online submission

- Thorough peer review

- Inclusion in PubMed and all major indexing services

- Maximum visibility for your research

Submit your manuscript at www.biomedcentral.com/submit 